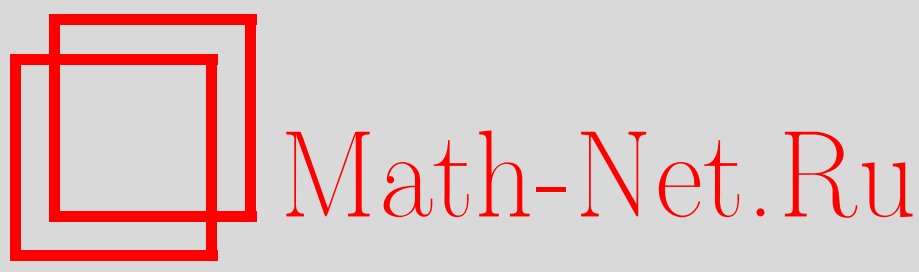

Е. И. Бережной, А. В. Новиков, О проблеме окаймления из теории дифференцирования интегралов, Изв. РАН. Сер. матем., 2002, том 66, выпуск 4, 3-26

DOI: https://doi.org/10.4213/im393

Использование Общероссийского математического портала Math-Net.Ru подразумевает, что вы прочитали и согласны с пользовательским соглашением

http://www . mathnet.ru/rus/agreement

Параметры загрузки:

IP : 18.209 .158 .208

26 апреля 2023 г., 08:53:20 
УДК 517.5

\author{
Е. И. Бережной, А. В. Новиков
}

\title{
О проблеме окаймления из теории дифференцирования интегралов
}

\begin{abstract}
Заданы пространство Лоренца и пространство Орлича с одинаковыми фундаментальными функциями. Строится дифференциальный базис, который дифференцирует все интегралы от функций из пространства Лоренца, но найдена функция из пространства Орлича, интеграл от которой построенный базис не дифференцирует. Сконструированные базисы позволяют отрицательно решить так называемую проблему окаймления для $p \in(1, \infty)$. В случае $p=1$ эта проблема положительно решена Морийоном $[1$, с. 186].

Библиография: 13 наименований.
\end{abstract}

\section{Введение}

Одна из основных задач теории дифференцирования интегралов дифференциальными базисами имеет следующий вид. Пусть $X, Y$ - два различных в каком-нибудь смысле пространства. Можно ли эти два пространства различить с помощью дифференщиальных базисов?, т.е. существует ли дифференщиальньй базис, который дифференцирует все интегралы от функций из $X$, но при этом найдется функция из $Y$, интеграл от которой данный базис не дифференцирует? Эта проблема еще далека от своего окончательного решения. Первый результат, имеющий отношение к этой проблеме, принадлежит Хейесу [1, с. 155], который показал, что можно различить пространства $L^{p}$ и $L^{q}$ при $p \neq q$, построив соответсвующий дифференциальный базис. Далее, А. Стоколос [2], [3] показал, что можно различить пространства Орлича, построив соответствующий дифференциальньй базис. Последние достижения по проблеме различения пар симметричных пространств с помощью дифференциальных базисов представлены в [4], где предложен новый способ построения дифференциальных базисов. Базисы, построенные этим способом, позволяют различать симметричные пространства с различньм поведением фундаментальных функций в нуле, как в случае, рассмотренном Хейесом и Стоколосом, и даже позволяют различать пространства Лоренца и Марцинкевича или пространства Лебега и Марцинкевича, у которых фундаментальные функции одинаковы. Наряду с задачей различения пары пространств можно рассматривать задачу различения пространства $X$ и набора пространств $\left\{Y_{\gamma}: \gamma \in \Gamma\right\}$ с помощью одного дифференциального базиса. Как показал Хейес [1, с. 165], существует дифференциальный базис, который дифференцирует $L^{\infty}$, но не дифференцирует $L^{p}$ при $1 \leqslant p<\infty$. Впрочем, справедливы непрерывные вложения $L^{\infty} \subset \exp (L) \subset \bigcap_{1 \leqslant p<\infty} L^{p}$, где $\exp (L)$ - пространство Орлича, построенное

Работа выполнена при финансовой поддержке РФФИ (грант № 02-01-00428).

(C) Е.И. БЕРЕЖной, А.В. Новиков, 2002 
по выпуклой функции $h(t)=\exp (|t|)-1$, и поэтому задачу различения $L^{\infty}$ и набора $\left\{L^{p}: 1 \leqslant p<\infty\right\}$ можно свести к задаче различения двух симметричных пространств $L^{\infty}$ и $\exp (L)$ с разным поведением в нуле фундаментальных функций. Поэтому последний результат Хейеса можно получить и из [4]. Позднее, в [5], был построен довольно простой дифференциальный базис, каждый элемент которого состоит только из двух квадратов и который дифференщирует $L^{\infty}$, а любое другое симметричное пространство $X \neq L^{\infty}$ не дифференщирует.

В настоящей работе решается задача различения с помощью дифференциальных базисов пары пространств Лоренца и Орлича, у которых фундаментальные функции одинаковы. Задача различения этой пары пространств оказалась значительно сложнее, чем задача различения пространств Лоренца и Марцинкевича или пространств Лебега и Марцинкевича с равными фундаментальными функциями. Базисы, построенные для решения задачи различения пары пространств Лоренца и Орлича, позволяют отрицательно решить известную проблему окаймления в случае пространства Лебега $L^{p}$ для $p \in(1, \infty)$. Для пространства $L^{1}$ проблема окаймления решена положительно Морийоном [1, с. 186 , добавление III].

\section{§ 1. Предварительные сведения}

Напомним некоторые определения, связанные с дифференциальными базисами. Эти определения можно найти в [1].

Дифференииальным базисом $B(t)$ в точке $t \in \mathbb{R}^{n}$ называется семейство содержаших $t$ ограниченных измеримых множеств положительной меры таких, что найдется по крайней мере одна последовательность $\left\{B_{k} \in B(t)\right\}$, удовлетворяющая условию $\operatorname{diam} B_{k} \rightarrow 0$. Дифференииальным базисом в $\mathbb{R}^{n}$ называется объединение указанных семейств $\mathbf{B}=\left\{\bigcup B(t): t \in \mathbb{R}^{n}\right\}$.

Классическими примерами дифференциальных базисов являются базисы в $\mathbb{R}^{n}$, обозначаемые обычно через $\mathbf{B}_{s}\left(\mathbb{R}^{n}\right), s=1,2, \ldots, n$, состояшие из всех прямоугольных параллелепипедов

$$
B=\left\{\left(t_{1}, \ldots, t_{n}\right) \in \mathbb{R}^{n}: \alpha_{i}<t_{i}<\alpha_{i}+\gamma_{i}, i=1, \ldots, n\right\},
$$

удовлетворяющих условию $\gamma_{j}=\gamma_{1}, j=1, \ldots, s ; \alpha_{i}, \gamma_{i} \in \mathbb{R}_{+}$.

Базис В, образованный открытыми множествами, называется базисом Буземана-Феллера (ВF-базисом), если из условий $B \in \mathbf{B}$ и $t \in B$ следует, что $B \in B(t)$. Смысл введения ВF-базисов состоит в том, что возникающие в теории дифференцирования по базисам вопросы измеримости легко решаются для ВF-базисов. Основные задачи и утверждения в теории дифференцирования интегралов заключаются в проверке почти всюду какого-либо факта. Поэтому естественно рассматривать дифференциальные базисы, определенные не для всех точек, а лишь почти всюду.

Определим теперь верхнюю и нижнюю производные интеграла от локально интегрируемой функции $f$ в точке $t \in \mathbb{R}^{n}$ относительно базиса $\mathbf{B}$ с помощью равенств

$$
\begin{aligned}
& \overline{D_{\mathbf{B}}(f, t)}=\sup \left\{\lim _{k \rightarrow \infty} \frac{1}{\mu\left(B_{k}\right)} \int_{B_{k}} f d \tau: \quad B_{k} \in B(t), \operatorname{diam} B_{k} \rightarrow 0\right\}, \\
& \underline{D_{\mathbf{B}}(f, t)}=\inf \left\{\frac{\lim }{k \rightarrow \infty} \frac{1}{\mu\left(B_{k}\right)} \int_{B_{k}} f d \tau: \quad B_{k} \in B(t), \operatorname{diam} B_{k} \rightarrow 0\right\} .
\end{aligned}
$$


Говорят, что базис $\mathbf{B}$ дифференцирует интеграл от $f$, если почти всюду выполнены равенства

$$
\overline{D_{\mathbf{B}}(f, t)}=\underline{D_{\mathbf{B}}(f, t)}=f(t) .
$$

Если $\mathbf{B}$ дифференцирует интеграл от любой функции $f$ из пространства $X$, то говорят, что базис В дифферениирует пространство $X$.

Если базис $\mathbf{B}$ дифференцирует $L^{\infty}$, то он называется плотностнылм.

Следуюшие классические разультаты явились фундаментом теории дифференцирования интегралов в $\mathbb{R}^{n}: \mathbf{B}_{s}$ дифференцирует $L\left(\log ^{+} L\right)^{n-s}$ (случай $s=n$ см. в [6], случай $s=1$ см. в [7], случай произвольного $s$ см. в [8]). То, что результат в случае $s=1$ невозможно усилить, показал в 1935 г. Сакс [9]. При других $s$ точность вышеупомянутых теорем была показана Мелеро [10].

Пусть В - дифференциальный базис. Важнейшую роль в теории дифференцирования интегралов дифференциальными базисами играет оператор максимальной функции, определяемый равенством

$$
M_{\mathbf{B}} f(t)=\sup \left\{\frac{1}{\mu(B)} \int_{B}|f(\tau)| d \tau: t \in B \in \mathbf{B}\right\} .
$$

ОПРЕДЕЛЕНИЕ. Пусть $\mathbf{B}$ - плотностной дифференциальньй базис и $M_{\mathbf{B}}-$ оператор максимальной функции, порожденный $\mathbf{B}$. Для дифференциального базиса $\mathbf{B}$ функиия окаймления $\psi_{\mathbf{B}}(\alpha)$ для $\alpha \geqslant 1$ определяется равенством

$$
\psi_{\mathbf{B}}(\alpha)=\sup _{D \subseteq Q}\left\{\mu(D)^{-1} \mu\left(\left\{\tau: M_{\mathbf{B}} \chi(D)(\tau)>\alpha^{-1}\right\}\right)\right\},
$$

где $\chi(D)=\chi(D, \tau)$-характеристическая функция множества $D$, причем аргумент $\tau$ будем часто опускать.

Проблема окаймления состоит в описании семейства тех пространств, в которых интегралы от функций дифференцируют базисы с данной функцией окаймления. В частности, если $\psi_{\mathbf{B}}(\alpha)$ - выпуклая функция, будет ли дифференциальный базис $\mathbf{B}$ дифференцировать пространство Орлича $L_{\psi_{\mathbf{B}}}$ ? На этот вопрос в случае $\psi_{\mathbf{B}}(\alpha)=$ $\alpha^{p}, 1 \leqslant p<\infty$, особо обрашалось внимание в [1, с. 163]. Морийон показал (см. [1, c. 186 , добавление III]), что если $\psi_{\mathbf{B}}(\alpha) \simeq \alpha$, то базис $\mathbf{B}$ дифференцирует $L^{1}$.

В настоящей работе показано, что дифференциальный базис с заданной функцией окаймления дифференцирует пространство Лоренца $\Lambda(\varphi)$, где функция $\varphi$ строится некоторым образом по функции $\psi_{\mathbf{B}}$. В частности, если $\psi_{\mathbf{B}}(\alpha)=\alpha^{p}, 1<p<$ $\infty$, то $\varphi(\alpha)=\alpha^{1 / p}$. Кроме того, для каждого $p \in(1, \infty)$ приведен пример дифференциального базиса $\mathbf{B}_{p}$, функция окаймления которого при больших $\alpha$ имеет вид $\psi_{\mathbf{B}}(\alpha) \simeq \alpha^{p}, 1<p<\infty$, и который не дифференцирует пространство Лебега $L^{p}$. Тем самым дан отрицательный ответ на вопрос из [1].

В дальнейшем потребуются две теоремы из теории дифференцирования интегралов дифференциальньми базисами.

Tеорема А [1, гл. III, теорема 1.1]. Пусть В является дифференциальнымм ВF-базисом. Два следующих свойства әквивалентны:

а) $\mathbf{B}$ есть плотностной базис;

b) для каждого $\alpha, 0<\alpha<1$, для каждой невозрастающей последовательности $\left\{A_{k}\right\}$ ограниченных измеримых множеств таких, что $\mu\left(A_{k}\right) \downarrow 0$, 
и для каждой невозрастающей последовательности $\left\{r_{k}\right\}$ действительных чисел таких, что $r_{k} \downarrow 0$, имеем

$$
\lim _{k \rightarrow \infty} \mu\left\{t: M_{k} \chi\left(A_{k}\right)(t)>\alpha\right\}=0
$$

əде

$$
M_{k} \chi\left(A_{k}\right)(t)=\sup \left\{\frac{1}{\mu(B)} \int_{B} \chi\left(A_{k}\right) d \tau: t \in B, \operatorname{diam}(B)<r_{k}, B \in \mathbf{B}\right\} .
$$

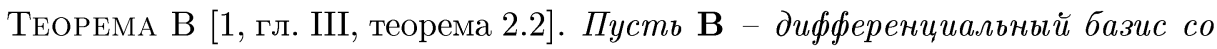
свойством плотности. Пусть $f \geqslant 0, f \in L^{1}$. Тогда три условия әквивалентни:

а) $\mathbf{B}$ дифферениирует $\int f(t) d t$;

b) для любого $\alpha>0$, для любой последовательности $\left\{f_{k}\right\}$ такой, что $0 \leqslant f_{k} \leqslant f, f_{k}(t) \downarrow 0$ почти всюду, и для любой числовой последовательности $\left\{r_{k}\right\}, r_{k} \downarrow 0$, выполняется соотношение

$$
\lim _{k \rightarrow \infty} \mu\left\{t: M_{k} f_{k}(t)>\alpha\right\}=0
$$

с) для любого $\alpha>0$, для любой невозрастающей последовательности $\left\{A_{k}\right\}$ ограниченных измеримых множеств таких, что $\mu\left(A_{k}\right) \downarrow 0$, и любой числовой последовательности $\left\{r_{k}\right\}$ действительных чисел такой, что $r_{k} \downarrow 0$, выполняется соотношение

$$
\lim _{k \rightarrow \infty} \mu\left\{t: M_{k}\left(f \chi\left(A_{k}\right)\right)(t)>\alpha\right\}=0 .
$$

Здесь

$$
M_{k} f(t)=\sup \left\{\frac{1}{\mu(B)} \int_{B}|f(\tau)| d \tau: t \in B, \operatorname{diam}(B)<r_{k}, B \in \mathbf{B}\right\} .
$$

Напомним некоторые сведения из теории симметричных пространств функций.

Пусть $Q_{0}$ - единичный куб в $\mathbb{R}^{n}$ с обычной мерой Лебега. Для $f: Q_{0} \rightarrow \mathbb{R}$ через $\lambda(f, \gamma)$ обозначим функцию распределения: $\lambda(f, \gamma)=\mu(\{\tau:|f(\tau)|>\gamma\})$, а через $f^{*}$ - ее перестановку в невозрастающем порядке: $f^{*}(\tau)=\inf \{s>0$ : $\lambda(f, s)<\tau\}$. Банахово пространство $X$, состоящее из измеримых функций, называется идеальныцм [11], если из $g \in X$, измеримости $f$ и выполнения п.в. неравенства $|f(\tau)| \leqslant|g(\tau)|$ следует, что $f \in X$ и $\|f|X\|\leqslant\| g| X\|$. Идеальное пространство называется симметричным [11], если для $f, g \in X$ из выполнения при всех $\gamma \in \mathbb{R}_{+}$ неравенства $\lambda(f, \gamma) \leqslant \lambda(g, \gamma)$ следует, что $\|f|X\|\leqslant\| g| X\|$. Как обычно, через $X^{\prime}$ обозначим пространство интегральных функционалов на $X$ с нормой

$$
\left\|g \mid X^{\prime}\right\|=\sup \left\{\int_{Q_{0}} g(\tau) f(\tau) d \tau: \quad\|f \mid X\| \leqslant 1\right\} .
$$

Для каждого симметричного пространства $X$ с помощью равенства $\psi(X, \tau)=$ $\{\|\chi(D) \mid X\|: \mu(D)=\tau\}$ определена фундаментальная функция. Эта функция 
не убывает, а $\tau^{-1} \psi(X, \tau)$ не возрастает. Классическими примерами симметричных пространств являются пространства Лебега $L^{p}$, Орлича $L_{h}$, Марцинкевича $M(\varphi)$, Лоренца $\Lambda(\varphi)$. Теория симметричных пространств функций подробно описана в [11], а симметричных пространств последовательностей - в [12]. Поскольку пространства Орлича, Лоренца и Марцинкевича будут использованы далее, опишем их более подробно. Обозначим через $V$ множество непрерывных выпуклых функций $h: \mathbb{R}_{+} \rightarrow \mathbb{R}_{+}$, для которых $h(0)=0, \lim _{\tau \rightarrow \infty} \frac{h(\tau)}{\tau}=\infty$. Пусть $h \in V$. Пространство Орлича $L_{h}$ состоит из тех измеримых функций, для которых конечна норма

$$
\left\|f \mid L_{h}\right\|=\inf \left\{\lambda>0: \int_{Q_{0}} h\left(\frac{|f(\tau)|}{\lambda}\right) d \tau \leqslant 1\right\}
$$

Отметим, что при рассмотрении пространства Орлича $L_{h}$ на $Q_{0}$ поведение функции $h$ в нуле не влияет на множество функций, из которых $L_{h}$ состоит.

Пусть задана функция $f:[0, a] \rightarrow \mathbb{R}_{+}$. Функиия растяжения $\rho_{f}: \mathbb{R}_{+} \rightarrow \mathbb{R}_{+}$ функции $f$ определяется с помощью равенства

$$
\rho_{f}(\lambda)=\sup _{0<\tau \leqslant \min \left\{a, \frac{a}{\lambda}\right\}} \frac{f(\lambda \tau)}{f(\tau)} .
$$

Прямо из определения следуют неравенства $f(\lambda \tau) \leqslant \rho_{f}(\lambda) f(\tau)$ и $\rho_{f}(\lambda \gamma) \leqslant$ $\rho_{f}(\lambda) \rho_{f}(\gamma)$.

Опишем теперь пространства Лоренца $\Lambda(\varphi)$ и Марцинкевича $M(\varphi)$. Обозначим через $W$ множество вогнутых функций $\varphi:[0,1] \rightarrow \mathbb{R}_{+}$, каждая из которых непрерывна, строго возрастает, $\varphi(0)=0$ и $\lim _{\tau \rightarrow 0} \tau^{-1} \varphi(\tau)=\infty$. С каждой функцией $\varphi \in W$ связана функция $\tilde{\varphi}(\tau)=\frac{\tau}{\varphi(\tau)}$. Операция $\tilde{\varphi}$ является инволюцией на класce $W$.

Пространство Лоренца $\Lambda(\varphi)$, построенное по $\varphi \in W$ (пространство Марцинкевича $M(\varphi))$, состоит из тех $f$, для которых конечна норма

$$
\begin{gathered}
\|f \mid \Lambda(\varphi)\|=\int_{0}^{1} f^{*}(\tau) d \varphi(\tau)=\int_{0}^{\infty} \varphi(\lambda(f, \tau)) d \tau \\
\left(\|f \mid M(\varphi)\|=\sup _{0<t<1} \frac{\varphi(t)}{t} \int_{0}^{t} f^{*}(\tau) d \tau=\sup _{D \subset Q_{0}}\left\{\frac{\varphi(\mu(D))}{\mu(D)} \int_{D}|f(\tau)| d \tau\right\}\right) .
\end{gathered}
$$

Если $\varphi(\tau)=\tau^{\theta}, 0<\theta<1$, то пространство Лоренца (Марцинкевича) в этом случае будем обозначать $\Lambda^{\theta}\left(M^{\theta}\right)$.

На пространстве Марцинкевича $M(\varphi)$ можно задать функционал

$$
N(f \mid M(\varphi))=\sup _{\alpha>0} \alpha \varphi(\lambda(f, \alpha))
$$

Нам потребуются две леммы из теории пространств Марцинкевича. 
Лемма 1. Пусть задано пространство Мариинкевича $M(\varphi)$. Для того чтобы при всех $f \in M(\varphi)$ выполнялись неравенства

$$
\frac{1}{c_{1}(\varphi)} N(f \mid M(\varphi)) \leqslant\|f \mid M(\varphi)\| \leqslant c_{1}(\varphi) N(f \mid M(\varphi)),
$$

необходимо и достаточно, чтобы выполнялось соотночение

$$
\sup _{0<t \leqslant 1}\left\{\frac{\varphi(t)}{t} \int_{0}^{t} \frac{d \tau}{\varphi(\tau)}\right\}<\infty
$$

В частности, если выполнено соотночение

$$
\rho_{\varphi}(2)<2,
$$

то выполнено соотношение (2) и, следовательно, выполнено (1).

ЛЕмма 2. Пусть заданы пространство Марцинкевича $M(\varphi)$ и число $\alpha_{0}>0$. Тогда для всех $f \in M(\varphi)$ выполняются неравенства

$$
\sup _{i \in \mathbb{Z}} 2^{i} \alpha_{0} \varphi\left(\lambda\left(f, 2^{i} \alpha_{0}\right)\right) \leqslant N(f \mid M(\varphi)) \leqslant 2 \sup _{i \in \mathbb{Z}} 2^{i} \alpha_{0} \varphi\left(\lambda\left(f, 2^{i} \alpha_{0}\right)\right) .
$$

Доказательство леммы 1 можно получить аналогично доказательству теоремы 5.3 из [11, с. 156], а доказательство леммы 2 легко получается прямо из определений.

Кроме того, нам потребуется одна лемма из теории пространств Лоренца.

Лемма А [11, гл. II, лемма 5.2]. Пусть задано пространство Лоренца $\Lambda(\varphi)$. Если выпуклый функционал $\Phi$ ограничен на всех характеристических функииях измеримых множеств, т.е. $\Phi(\chi(A)) \leqslant c\|\chi(a) \mid \Lambda(\varphi)\|$, то для всех обобщенно конечнозначных функций $f \in \Lambda(\varphi)$ выполнено неравенство $\Phi(f) \leqslant$ $2 c\|f \mid \Lambda(\varphi)\|$.

Хорошо известна двойственность пространств Лоренща и Марцинкевича [11]:

$$
\Lambda(\varphi)^{\prime}=M(\tilde{\varphi}), \quad M(\varphi)^{\prime}=\Lambda(\tilde{\varphi})
$$

Для фундаментальных функций пространств Лебега, Орлича, Лоренца, Марцинкевича верны равенства

$$
\psi\left(L^{p}, t\right)=t^{1 / p}, \quad \psi\left(L_{h}, t\right)=\frac{1}{h^{-1}(1 / t)}, \quad \psi(\Lambda(\varphi), t)=\psi(M(\varphi), t)=\varphi(t) .
$$

Пусть задана функция $\varphi \in W$, по которой построено пространство Лоренца $\Lambda(\varphi)$. Опишем, как по $\varphi \in W$ построить пространство Орлича $L_{h_{\varphi}}$, у которого фундаментальная функция совпадает с $\varphi$. Определим функцию $h_{\varphi}$ из равенства

$$
h_{\varphi}^{-1}(\tau)=\left\{\begin{array}{lll}
\frac{1}{\varphi\left(\frac{1}{\tau}\right)} & \text { при } \tau \geqslant 1, \\
\frac{\tau}{\varphi(1)} & \text { при } \tau \in[0,1) .
\end{array}\right.
$$


Из неравенства $\frac{1}{\varphi(1)} \geqslant \frac{\varphi^{\prime}(1)}{\varphi^{2}(1)}=\left.\left(\frac{1}{\varphi(\tau)}\right)^{\prime}\right|_{\tau=1}$ следует, что $h_{\varphi}-$ выпуклая функция. Очевидно, что $h_{\varphi}(0)=0$, а из $\varphi \in W$ следует, что $\lim _{\tau \rightarrow \infty} \frac{h_{\varphi}(\tau)}{\tau}=\infty$. Построим по $h_{\varphi}$ пространство Орлича $L_{h_{\varphi}}$. Тогда из равенства (5) следует, что фундаментальные функции пространств $L_{h_{\varphi}}$ и $\Lambda(\varphi)$ совпадают. Наоборот, если задана функция $h \in V$, то, полагая $\varphi_{h}(0)=0$ и определяя функцию $\varphi_{h}$ при $\tau \in(0,1]$ равенством

$$
\varphi_{h}(\tau)=\frac{1}{h^{-1}(1 / \tau)}
$$

согласно (5) и $h \in V$ получим, что $\varphi_{h} \in W$ и фундаментальные функции пространств $L_{h}$ и $\Lambda\left(\varphi_{h}\right)$ совпадают.

\section{§ 2. Различение пространств Орлича и Лоренца с помощью дифференциального базиса}

В настоящем параграфе покажем, что при некоторых условиях на фундаментальную функцию пространства Лоренща существует плотностной дифференциальный базис $\mathbf{B}_{\varphi}$, который различает пространство Лоренца $\Lambda(\varphi)$ и пространство Орлича $L_{h_{\varphi}}$, т.е. дифференщиальный базис $\mathbf{B}_{\varphi}$ дифференщирует пространство Лоренща $\Lambda(\varphi)$ и не дифференцирует пространство Орлича $L_{h_{\varphi}}$. Пример дифференциального базиса $\mathbf{B}_{\varphi}$ строится конструктивно. Аналогично сконструированный дифференциальный базис будет использован и при отрицательном решении проблемы окаймления для $L^{p}$ при $p \in(1, \infty)$. Для простоты и наглядности изложения будем строить дифференциальные базисы на плоскости, т.е. в $\mathbb{R}^{2}$.

Зафиксируем квадрат $Q_{0}$, числа $n, l \in \mathbb{N}$ и набор $\bar{m}=\left\{\bar{m}_{0}, \bar{m}_{1}, \ldots, \bar{m}_{l}\right\}$, где $\bar{m}_{i} \in \mathbb{N}$, причем будем предполагать, что для всех $j=0,1, \ldots, l-1$ с некоторой костантой $c_{0}$ выполнено соотношение

$$
\sum_{i=0}^{j} \bar{m}_{i} \leqslant c_{0} \bar{m}_{j+1} .
$$

Положим

$$
k=\min \left\{j: \sum_{i=0}^{l} 2^{i} \bar{m}_{i} \leqslant 2^{2 j}\right\} .
$$

Для фиксированных $l, k, \bar{m}$ определим набор $m=\left\{m_{0}, m_{1}, \ldots, m_{l}\right\}$ с помощью формул

$$
\begin{gathered}
m_{j}=\bar{m}_{j}, \quad j=0,1, \ldots, l-1, \\
m_{l}=\left[2^{2 k-l}-2^{-l} \sum_{i=0}^{l-1} 2^{i} \bar{m}_{i}\right]
\end{gathered}
$$

где $[x]$ - целая часть числа $x$.

Лемма 3. Для определенного равенством (10) числа $m_{l}$ справедливы неравенства

$$
\bar{m}_{l} \leqslant m_{l}<\left(4+\frac{3}{2} c_{0}\right) \bar{m}_{l} .
$$


ДокАЗАТЕЛЬСТво. Левая часть неравенства (11) легко следует из (10), а правая часть следует из (9) и (10):

$$
\begin{aligned}
m_{l} & \leqslant 2^{2 k-l}-2^{-l} \sum_{i=0}^{l-1} 2^{i} \bar{m}_{i} \leqslant 4 \cdot 2^{-l} \sum_{i=0}^{l} 2^{i} \bar{m}_{i}-2^{-l} \sum_{i=0}^{l-1} 2^{i} \bar{m}_{i} \\
& =3 \sum_{i=0}^{l-1} 2^{i-l} \bar{m}_{i}+4 \bar{m}_{l} \leqslant \frac{3}{2} \sum_{i=0}^{l-1} \bar{m}_{i}+4 \bar{m}_{l} \leqslant \bar{m}_{l}\left(4+\frac{3}{2} c_{0}\right) .
\end{aligned}
$$

Лемма доказана.

Из леммы 3 и неравенств (8) и (11) следуют соотношения

$$
\sum_{i=0}^{j} m_{i} \leqslant c_{0} m_{j+1}, \quad j=0,1, \ldots, l-1 .
$$

Изложим способ построения некоторого набора множеств из квадрата $Q_{0}$. Этот прием будет неоднократно использоваться далее, и поэтому мы назовем его основной конструкцией.

Разобьем исходный квадрат $Q_{0}$ на $n^{2}$ равных квадратов $Q_{n}(j)$, а затем каждый из квадратов $Q_{n}(j), j=1,2, \ldots, n^{2}$, разобьем на $2^{2 k}$ равных квадратов, которые занумеруем каким-либо фиксированным способом $Q_{n}(j, i), i=1,2, \ldots, 2^{2 k}$. Для каждого из квадратов $Q_{n}(j)$ проделаем следующую стандартную процедуру, которую продемонстрируем для квадрата $Q_{n}(1)$. Из квадратов $Q_{n}(1, i)$ построим некоторый набор пачек квадратов. Пачка нулевого уровня $P(0,0)$ будет только одна, и она состоит из первых $m_{0}$ квадратов $Q_{n}(1, i)$. Пачек первого уровня будет две: $P(1,0), P(1,1)$, причем $P(1,0)$ состоит из $m_{1}$ квадратов $Q_{n}(1, i)$, не принадлежаших $P(0,0)$, а $P(1,1)$ состоит из $m_{1}$ квадратов $Q_{n}(1, i)$, не принадлежащих $P(0,0)$ и $P(1,0)$. Пачек $s$-го уровня, $0 \leqslant s \leqslant l$, будет $2^{s}$ штук: $P(s, 0), P(s, 1), \ldots, P\left(s, 2^{s}-1\right)$, причем каждая из них состоит из $m_{s}$ квадратов, которые принадлежат только этой пачке.

Лемма 4. Число квадратов из набора $Q_{n}(1, i), i=1,2, \ldots, 2^{2 k}$, не попавших ни в одну из пачек всех уровней, не превышает числа $2^{l}$.

ДокАЗАТЕльСТво. Число квадратов в наборе $Q_{n}(1, i)$ есть $2^{2 k}$. Поэтому, используя конструкцию пачек, получим

$$
\begin{aligned}
2^{2 k}-\sum_{i=0}^{l-1} 2^{i} m_{i}-2^{l} m_{l} & =2^{2 k}-\sum_{i=0}^{l-1} 2^{i} \bar{m}_{i}-2^{l}\left[2^{2 k-l}-2^{-l} \sum_{i=0}^{l-1} 2^{i} \bar{m}_{i}\right] \\
& \leqslant 2^{2 k}-\sum_{i=0}^{l-1} 2^{i} \bar{m}_{i}-2^{l}\left(2^{2 k-l}-2^{-l} \sum_{i=0}^{l-1} 2^{i} \bar{m}_{i}-1\right) \leqslant 2^{l}
\end{aligned}
$$

Лемма доказана.

Отметим еше одно важное соотношение

$$
\frac{1}{2+c_{0}} \leqslant \frac{m_{l}}{2^{2 k-l}}<1
$$


которое следует из неравенства

$$
2^{2 k} \leqslant 2^{l} \sum_{i=0}^{l} m_{i}+2^{l}=2^{l}\left(1+\left(1+c_{0}\right) m_{l}\right) \leqslant 2^{l}\left(2+c_{0}\right) m_{l} .
$$

Образуем теперь множества $U_{n}(1, s), s=1,2, \ldots, 2^{l}$, которые будут составлять набор $U_{n}(1)=\bigcup_{s=1}^{2^{l}} U_{n}(1, s)$. Множество $U_{n}(1, s)$ содержит ровно по одной пачке квадратов каждого уровня. Если $i=0,1, \ldots, l$ и число $s$ удовлетворяет неравенству $p 2^{l-i}<s \leqslant(p+1) 2^{l-i}$, то множество $U_{n}(1, s)$ содержит все квадраты пачки $P(i, p)$.

Элементы набора $U_{n}(1)$ обладают различными свойствами, которые отражены в следующей лемме.

ЛЕмма 5. Для $i=0,1, \ldots, l$ все квадраты из фиксированной пачки $i$-го уровня принадлежат $2^{l-i}$ множествам из набора $U_{n}(1)$. Для любого множсества $U_{n}(1, s) \in U_{n}(1)$ выполнено равенство

$$
\mu\left(U_{n}(1, s)\right)=\mu\left(Q_{n}(1,1)\right) \sum_{i=0}^{l} m_{i}
$$

Справедливы неравенства

$$
\begin{aligned}
& \mu\left(Q_{n}(1,1)\right)|I| m_{l} \leqslant \mu\left(\bigcup_{p \in I} U_{n}(1, p)\right) \leqslant \mu\left(Q_{n}(1,1)\right)|I| \sum_{i=0}^{l} m_{i} \\
& \frac{1}{1+c_{0}} \sum_{i \in I} \mu\left(U_{n}(1, i)\right) \leqslant \mu\left(\bigcup_{i \in I} U_{n}(1, i)\right) \leqslant \sum_{i \in I} \mu\left(U_{n}(1, i)\right)
\end{aligned}
$$

ДокАЗАТЕЛЬСтво первых двух утверждений леммы и неравенства (14) следует из построения. Неравенство (15) следует из (12). Лемма доказана.

С каждым квадратом $Q_{n}(1)$ и набором $U_{n}(1)$ свяжем оператор максимальной функции

$$
M_{U_{n}(1)} f(t)=\sup \left\{\frac{1}{\mu\left(U_{n}(1, s)\right)} \int_{U_{n}(1, s)}|f(\tau)| d \tau: t \in U_{n}(1, s) \in U_{n}(1)\right\},
$$

набор множеств

$$
R_{U_{n}(1)}(s)=\bigcup_{i=0}^{2^{s}-1} P(s, i), \quad s=0,1, \ldots, l,
$$

и функцию $f_{U_{n}(1)}$, определенную равенством

$$
f_{U_{n}(1)}(t)=\frac{m_{l}}{l} \sum_{i=0}^{l-1} \frac{1}{m_{i}} \sum_{q=0}^{2^{i}-1} \sum_{Q_{n}(1, p) \in P(i, q)} \chi\left(Q_{n}(1, p)\right) .
$$

Наша ближайшая цель - получить эффективную оценку оператора максимальной функции. Следующая лемма является ключевой для характеризации дифференциальных свойств базисов, которые будут рассмотрены в настоящей работе. 
ЛЕмма 6. Пусть задан квадрат $Q_{n}(1)$, число $l$ и набор $\bar{m}=\left(\bar{m}_{0}, \ldots, \bar{m}_{l}\right)$, удовлетворяющий соотношению (8), по которому построен новый набор натуральных чисел $m=\left(m_{0}, \ldots, m_{l}\right)$. Пусть задана функиия $\varphi \in W$, для которой $\varphi(1)=1$ и по которой построено пространство Лоренца $\Lambda(\varphi)$. Пусть $f \in \Lambda(\varphi), f(t) \geqslant 0$ почти всюду. Пусть задано $\alpha>0$. Положим

$D(f, \alpha)=\left\{t: M_{U_{n}(1)} f(t)>\alpha\right\}=\left\{\bigcup U_{n}(1, s): \frac{1}{\mu\left(U_{n}(1, s)\right)} \int_{U_{n}(1, s)} f(\tau) d \tau>\alpha\right\}$.

Тогда справедливо соотношение

$$
\mu(D(f, \alpha)) \leqslant \frac{1}{\alpha} \int_{D(f, \alpha)} \xi(\alpha, f, \tau) f(\tau) d \tau,
$$

где $\xi(\alpha, f, \tau) \geqslant 0$ всюду, и выполнены следующие неравенства:

1) если $p=-1,-2, \ldots$, mo

$$
\mu\left(\left\{\tau: \xi(\alpha, f, \tau) \geqslant 2^{p}\right\}\right) \leqslant \mu(D(f, \alpha))
$$

2) если $p=0,1, \ldots, l$, mo

$$
\mu\left(\left\{\tau: \xi(\alpha, f, \tau) \geqslant 2^{p}\right\}\right) \leqslant\left(1+c_{0}\right) \mu(D(f, \alpha)) \frac{m_{l-p}}{m_{l} 2^{p}} ;
$$

3) если $p=l+1, l+2, \ldots$, mo

$$
\mu\left(\left\{\tau: \xi(\alpha, f, \tau) \geqslant 2^{p}\right\}\right)=0 .
$$

ДоКАЗАТЕЛЬСТВо. Положим $I=\left\{s: U_{n}(1, s) \in D(f, \alpha)\right\}$. Для каждого $Q_{n}(1, i) \subseteq D(f, \alpha)$ через $d_{i}$ обозначим кратность $Q_{n}(1, i)$, с которой он входит в $D(f, \alpha)$. Тогда справедлива оценка

$$
\begin{aligned}
\mu(D(f, \alpha)) & =\mu\left(\bigcup_{s \in I} U_{n}(1, s)\right) \leqslant \sum_{s \in I} \mu\left(U_{n}(1, s)\right)<\frac{1}{\alpha} \sum_{s \in I} \int_{U_{n}(1, s)} f(\tau) d \tau \\
& =\frac{1}{\alpha} \sum_{Q_{n}(1, i) \subseteq D(f, \alpha)} d_{i} \int_{Q_{n}(1, i)} f(\tau) d \tau \\
& =\frac{1}{\alpha} \int_{D(f, \alpha)} \sum_{Q_{n}(1, i) \subseteq D(f, \alpha)} d_{i} \chi\left(Q_{n}(1, i)\right) f(\tau) d \tau .
\end{aligned}
$$

Положим

$$
\xi(\alpha, f, \tau)=\sum_{Q_{n}(1, i) \subseteq D(f, \alpha)} d_{i} \chi\left(Q_{n}(1, i), \tau\right)
$$

и оценим для каждого $p \in \mathbb{Z}$ величину $c(p)=\mu\left(\left\{t: \xi(\alpha, f, t) \geqslant 2^{p}\right\}\right)$. Очевидно, что при $p=-1,-2, \ldots$ верно неравенство

$$
c(p) \leqslant \mu(D(f, \alpha))
$$


Если $p=l+1, l+2, \ldots$, то $\left\{\tau: \xi(\alpha, f, \tau) \geqslant 2^{p}\right\}=\varnothing$, и поэтому

$$
\mu\left(\left\{\tau: \xi(\alpha, f, \tau) \geqslant 2^{p}\right\}\right)=0 .
$$

Оценим теперь $c(p)$ при $p=0,1, \ldots, l$. Обозначим через $|I|$ число множеств в наборе $D(f, \alpha)=\bigcup_{i \in I} U_{n}(1, i)$. Очевидно, что $c(p)=0$ при $2^{p}>|I|$. Если же $2^{p} \leqslant|I|$, то вместо оценки величины $c(p)$ оценим меру большего множества $W(p)$, состоящего из всех квадратов, входящих в $\bigcup_{i \in I} U_{n}(1, i)$ с кратностью, большей или равной $2^{p}$. Для этого сначала отметим, что если $Q_{n}(1, i) \subseteq W(p)$, то вся пачка, содержашая $Q_{n}(1, i)$, входит в $W(p)$. Поэтому можно сначала сосчитать число пачек, квадраты из которых могут входить в $W(p)$, а затем умножить это число пачек на число элементов, содержашихся в одной пачке.

Согласно первому утверждению леммы 5 во множество $W(p)$ попадут только элементы пачек, находяшихся на уровне $j, 0 \leqslant j \leqslant l-p$. Поскольку в рассмотрении участвует всего $|I|$ множеств, то число $k(j)$ пачек уровня $j, j \leqslant l-p$, допускает элементарную оценку

$$
k(j) \leqslant \min \left\{\left[\frac{|I|}{2^{p}}\right], 2^{j}\right\},
$$

где $[x]$ - целая часть числа $x$. Поэтому из (12) и (21) следует неравенство

$$
\begin{aligned}
c(p) & \leqslant \mu(W(p)) \leqslant \mu\left(Q_{n}(1,1)\right) \sum_{j=0}^{l-p} k(j) m_{j} \\
& \leqslant \mu\left(Q_{n}(1,1)\right)\left[\frac{|I|}{2^{p}}\right] \sum_{j=0}^{l-p} m_{j} \leqslant\left(1+c_{0}\right)\left[\frac{|I|}{2^{p}}\right] m_{l-p} \mu\left(Q_{n}(1,1)\right) .
\end{aligned}
$$

Согласно лемме 5 окончательно будем иметь

$$
\begin{aligned}
c(p) & \leqslant \mu(W(p)) \leqslant\left(1+c_{0}\right)\left[\frac{|I|}{2^{p}}\right] m_{l-p} \mu\left(Q_{n}(1,1)\right) \\
& \leqslant\left(1+c_{0}\right) \frac{|I| m_{l} \mu\left(Q_{n}(1,1)\right)}{m_{l} 2^{p}} m_{l-p} \leqslant\left(1+c_{0}\right) \mu(D(f, \alpha)) \frac{m_{l-p}}{m_{l} 2^{p}} .
\end{aligned}
$$

Из последнего неравенства, (19) и (20) следует утверждение леммы.

ЛЕмма 7. Для любого множества $U_{n}(1, i)$, построенного с помощью основной конструкции на квадрате $Q_{n}(1)$, справедливо неравенство

$$
\frac{1}{\mu\left(U_{n}(1, i)\right)} \int_{U_{n}(1, i)} f_{U_{n}(1)}(\tau) d \tau \geqslant \frac{1}{1+c_{0}} .
$$

ДОКАЗАТЕЛЬСТВо. Согласно построению будем иметь

$$
\frac{1}{\mu\left(U_{n}(1, i)\right)} \int_{U_{n}(1, i)} f_{U_{n}(1)}(\tau) d \tau \geqslant \frac{m_{l}}{\sum_{i=0}^{l} m_{i}} \geqslant \frac{m_{l}}{\left(1+c_{0}\right) m_{l}}=\frac{1}{1+c_{0}} .
$$

Лемма полностью доказана. 
С каждым из квадратов $Q_{n}(j), j=2, \ldots, n^{2}$, проделаем процедуру, аналогичную построению набора $U_{n}(1)$ для квадрата $Q_{n}(1)$, и получим наборы $U_{n}(j)$, максимальные функции $M_{U_{n}(j)}$ и функции $f_{U_{n}(j)}$. Объединим все элементы наборов $U_{n}(j)$ в новый набор множеств

$$
U_{n, l, m}=\bigcup_{j=1}^{n^{2}} U_{n}(j) .
$$

По набору $U_{n, l, m}$ в квадрате $Q_{0}$ построим оператор максимальной функции

$$
M_{n, l, m} f(t)=\sup \left\{\frac{1}{\mu\left(U_{n}(j, i)\right)} \int_{U_{n}(j, i)}|f(\tau)| d \tau: t \in U_{n}(j, i) \in U_{n, l, m}\right\} .
$$

Очевидно, что справедливы равенства

$$
M_{n, l, m} f(t)=\sum_{j=1}^{n^{2}} M_{U_{n}(j)} f(t)=\max _{j=1, \ldots, n^{2}} M_{U_{n}(j)} f(t) .
$$

Кроме того, по набору $U_{n, l, m}$ и функциям $f_{U_{n}(j)}$ в квадрате $Q_{0}$ построим новую функцию

$$
f_{n, l, m}(t)=\sum_{j=1}^{n^{2}} f_{U_{n}(j)}=\max _{j=1, \ldots, n^{2}} f_{U_{n}(j)}
$$

а по множествам $R_{U_{n}(j)}$ - новые множества

$$
R_{n, l, m}(s)=\bigcup_{j=1}^{n^{2}} R_{U_{n}(j)}(s), \quad s=0,1, \ldots, l .
$$

Из построения, неравенства (13) и леммы 4 получим, что для множеств $R_{n, l, m}(s)$ выполнены соотношения

$$
\begin{aligned}
\mu(\{t: & \left.\left.\frac{1}{\mu(U)} \int_{U} \chi\left(R_{n, l, m}(s), \tau\right) d \tau \geqslant \frac{m_{s}}{\left(1+c_{0}\right) m_{l}}, \quad t \in U \in U_{n, l, m}\right\}\right) \\
= & \left(1-2^{l-2 k}\right) \frac{\mu\left(Q_{0}\right)}{\mu\left(R_{n, l, m}(s)\right)} \mu\left(R_{n, l, m}(s)\right)=\left(1-2^{l-2 k}\right) \frac{2^{2 k}}{2^{s} m_{s}} \mu\left(R_{n, l, m}(s)\right) \\
\quad= & \left(1-2^{l-2 k}\right) \frac{2^{2 k-l} 2^{l-s}}{m_{s}} \mu\left(R_{n, l, m}(s)\right) \geqslant\left(1-2^{l-2 k}\right) \frac{m_{l} 2^{l-s}}{m_{s}} \mu\left(R_{n, l, m}(s)\right) .
\end{aligned}
$$

Применяя элементарное неравенство

$$
2^{2 k-l}>m_{l}>\frac{1}{c_{0}} \sum_{i=0}^{l-1} m_{i}>\frac{l}{c_{0}},
$$

получим, что для множеств $R_{n, l, m}(s)$ справедливы важные неравенства

$$
\begin{gathered}
\mu\left(\left\{t: \frac{1}{\mu(U)} \int_{U} \chi\left(R_{n, l, m}(s), \tau\right) d \tau \geqslant \frac{m_{s}}{\left(1+c_{0}\right) m_{l}}, \quad t \in U \in U_{n, l, m}\right\}\right) \\
\geqslant\left(1-\frac{c_{0}}{l}\right) \frac{m_{l} 2^{l-s}}{m_{s}} \mu\left(R_{n, l, m}(s)\right) .
\end{gathered}
$$

Из леммы 7 непосредственно получим следующую лемму. 
ЛЕмма 8. Для любого множества $U \in U_{n, l, m}$ справедливо неравенство

$$
\frac{1}{\mu(U)} \int_{U} f_{n, l, m}(\tau) d \tau \geqslant \frac{1}{1+c_{0}}
$$

Пусть задана функция $\varphi \in W$, по которой согласно формуле (6) построена функция $h_{\varphi}$. В следующей лемме дана эффективная оценка нормы функции $f_{n, l, m}(t)$ в пространстве Орлича $L_{h_{\varphi}}$.

ЛЕмма 9. Пусть задана строго монотонная функиия $\varphi \in W$, для которой $\varphi(1)=1$. Пусть, кроме того, для всех $i=0,1, \ldots, l$ с некоторой константой $c_{2}$ выполнено соотношение

$$
\frac{m_{i}}{2^{l-i} m_{l}} \leqslant c_{2} \varphi^{-1}\left(\frac{m_{i}}{m_{l}}\right)
$$

Тогда справедливо неравенство

$$
\left\|f_{n, l, m} \mid L_{h_{\varphi}}\right\| \leqslant \inf \left\{\lambda>0: \rho_{\varphi^{-1}}\left(\frac{1}{\lambda}\right) \leqslant \frac{1}{c_{2} l \rho_{\varphi^{-1}}\left(\frac{1}{l}\right)}\right\}
$$

ДоКАЗАТЕЛЬСТВо. Согласно построению будем иметь

$$
\begin{aligned}
\left\|f_{n, l, m} \mid L_{h_{\varphi}}\right\| & =\inf \left\{\lambda>0: \int_{Q_{0}} h_{\varphi}\left(\frac{f_{n, l, m}(\tau)}{\lambda}\right) d \tau \leqslant 1\right\} \\
& =\inf \left\{\lambda>0: n^{2} \int_{Q_{n}(1)} h_{\varphi}\left(\frac{f_{U_{n}(1)}(\tau)}{\lambda}\right) d \tau \leqslant 1\right\} \\
& =\inf \left\{\lambda>0: n^{2} \sum_{i=0}^{l-1} h_{\varphi}\left(\frac{m_{l}}{\lambda l m_{i}}\right) 2^{i} m_{i} \mu\left(Q_{n}(1,1)\right) \leqslant 1\right\} \\
& =\inf \left\{\lambda>0: n^{2} \sum_{i=0}^{l-1} h_{\varphi}\left(\frac{m_{l}}{\lambda l m_{i}}\right) 2^{i} m_{i} \frac{\mu\left(Q_{0}\right)}{n^{2} 2^{2 k}} \leqslant 1\right\} \\
& =\inf \left\{\lambda>0: \sum_{i=0}^{l-1} h_{\varphi}\left(\frac{m_{l}}{\lambda l m_{i}}\right) 2^{i} m_{i} \frac{1}{2^{2 k}} \leqslant 1\right\} \\
& =\inf \left\{\lambda>0: \sum_{i=0}^{l-1} \frac{1}{\varphi^{-1}\left(\frac{l \lambda m_{i}}{m_{l}}\right)} 2^{i} m_{i} \frac{1}{2^{2 k}} \leqslant 1\right\} \\
& =\inf \left\{\lambda>0: \sum_{i=0}^{l-1} \frac{1}{\varphi^{-1}\left(\frac{l \lambda m_{i}}{m_{l}}\right)} \frac{m_{i}}{2^{l-i} m_{l}} \frac{m_{l}}{2^{2 k-l}} \leqslant 1\right\} \\
& \leqslant \inf \left\{\lambda>0: c_{2} \sum_{i=0}^{l-1} \frac{\varphi^{-1}\left(\frac{m_{i}}{m_{l}}\right)}{\varphi^{-1}\left(\frac{l \lambda m_{i}}{m_{l}}\right)} \frac{m_{l}}{2^{2 k-l}} \leqslant 1\right\} \\
& \leqslant \inf \left\{\lambda>0: c_{2} \sum_{i=0}^{l-1} \rho_{\varphi^{-1}}\left(\frac{1}{\lambda}\right) \rho_{\varphi^{-1}}\left(\frac{1}{l}\right) \frac{m_{l}}{2^{2 k-l}} \leqslant 1\right\}
\end{aligned}
$$




$$
\leqslant \inf \left\{\lambda>0: c_{2} l \rho_{\varphi^{-1}}\left(\frac{1}{\lambda}\right) \rho_{\varphi^{-1}}\left(\frac{1}{l}\right) \frac{m_{l}}{2^{2 k-l}} \leqslant 1\right\}
$$

Из (13) следует, что $\frac{m_{l}}{2^{2 k-l}} \leqslant 1$. Поэтому

$$
\left\|f_{n, l, m} \mid L_{h_{\varphi}}\right\| \leqslant \inf \left\{\lambda>0: l \rho_{\varphi^{-1}}\left(\frac{1}{\lambda}\right) \rho_{\varphi^{-1}}\left(\frac{1}{l}\right) \leqslant c_{2}^{-1}\right\}
$$

Лемма полностью доказана.

Следуюшая лемма нам потребуется для определения наборов натуральных чисел при построении базиса, дифференцируюшего $\Lambda(\varphi)$, и “плохой” функции из пространства Орлича $L_{h_{\varphi}}$, интеграл от которой этот базис не будет дифференцировать.

Лемма 10. Пусть задана строго монотонная функиия $\varphi \in W$, для которой $\varphi(1)=1$. Пусть $i \in \mathbb{N}$. Тогда уравнение

$$
\varphi\left(2^{-i} t\right)=t
$$

имеет единственное решение $t_{i}$ при каждом $i \in \mathbb{N}$, причем при всех $i \in \mathbb{N}$ справедливо неравенство

$$
t_{i+1} \leqslant \rho_{\varphi}\left(\frac{1}{2}\right) t_{i}
$$

и, следовательно, справедливо неравенство

$$
t_{i} \leqslant\left(\rho_{\varphi}\left(\frac{1}{2}\right)\right)^{i}
$$

ДокАЗАТЕЛЬСТВо. Поскольку функция $\varphi \in W$ строго возрастает, то функция $\frac{\varphi\left(2^{-i} t\right)}{t}$ удовлетворяет соотношениям $\frac{\varphi\left(2^{-i}\right)}{1}=\varphi\left(2^{-i}\right)<1$ и $\lim _{t \rightarrow \infty} \frac{\varphi\left(2^{-i} t\right)}{t}=\infty$. Поэтому уравнение (28) имеет единственное решение. Поскольку при $t>0$ верно неравенство $\varphi\left(2^{-i} t\right)>\varphi\left(2^{-i-1} t\right)$, то $t_{i+1}<t_{i}$ и для получения оценки $(29)$ можно воспользоваться цепочкой неравенств

$$
\begin{aligned}
t_{i+1} & =\varphi\left(2^{-i-1} t_{i+1}\right)=\varphi\left(\frac{1}{2} 2^{-i} t_{i+1}\right) \leqslant \rho_{\varphi}\left(\frac{1}{2}\right) \varphi\left(2^{-i} t_{i+1}\right) \\
& <\rho_{\varphi}\left(\frac{1}{2}\right) \varphi\left(2^{-i} t_{i}\right)=\rho_{\varphi}\left(\frac{1}{2}\right) t_{i} .
\end{aligned}
$$

Неравенство (30) следует из (29) и равенства $t_{0}=1$. Лемма доказана.

Теперь можно перейти к доказательству основной теоремы этого параграфа. 
TEOPEMA 1. Пусть задана строго монотонная функиия $\varphi \in W$, для которой $\varphi(1)=1$. Пусть выполнены соотношение (3) и следующие условия:

$$
\begin{gathered}
\rho_{\varphi}\left(\frac{1}{2}\right)=q<1, \\
\rho_{\varphi^{-1}}(2)<\infty, \\
\lim _{\alpha \rightarrow \infty} \alpha \rho_{\varphi^{-1}}\left(\frac{1}{\alpha}\right)=0 .
\end{gathered}
$$

Тогда существует дифференциальныи ВF-базис $\mathbf{B}_{\varphi}$, который дифференцирует пространство Лоренца $\Lambda(\varphi)$, но не дифференцирует пространство $L_{h_{\varphi}}$.

ДокАЗАТЕльСТво. Выберем сначала согласно (31) убывающую последовательность положительных чисел $\left\{\lambda_{i}\right\}$ таких, чтобы выполнялось неравенство

$$
\sum_{i=1}^{\infty} \lambda_{i}<\frac{1-\rho_{\varphi}\left(\frac{1}{2}\right)}{2} .
$$

Затем выберем возрастающую последовательность натуральных чисел $\left\{l_{j}\right\}_{j=1}^{\infty}$ таких, чтобы выполнялись соотношения

$$
\rho_{\varphi^{-1}}\left(\frac{1}{\lambda_{i}}\right) \leqslant \frac{1}{c_{2} l_{i} \rho_{\varphi^{-1}}\left(\frac{1}{l_{i}}\right)}
$$

где $c_{2}=2 \rho_{\varphi^{-1}}\left(8+3 \frac{\rho_{\varphi}\left(\frac{1}{2}\right)}{1-\rho_{\varphi}\left(\frac{1}{2}\right)}\right)$. Такой выбор возможен в силу выполнения условия (33). Для каждого $l_{j}$ определим набор чисел $\bar{m}(j)=\left\{\bar{m}_{0}(j), \bar{m}_{1}(j), \ldots, \bar{m}_{l_{j}}(j)\right\}$ из соотношений

$$
\bar{m}_{i}(j)=\left[\frac{t_{l_{j}-i}}{t_{l_{j}}}\right], \quad i=0,1, \ldots, l_{j}
$$

где $t_{l_{j}-i}-$ корень уравнения (28). Покажем, что для построенного таким образом набора выполнено условие (8). Действительно, из элементарного неравенства $[\alpha x] \leqslant \alpha[x], 0 \leqslant \alpha \leqslant 1, x \geqslant 0$, где $[x]$ - целая часть числа $x$, и определения чисел $\bar{m}_{i}(j)$ для каждого $p=0,1, \ldots, l_{j}-1$ получим

$$
\begin{aligned}
\sum_{i=0}^{p} \bar{m}_{i}(j) & =\sum_{i=0}^{p}\left[\frac{t_{l_{j}-i}}{t_{l_{j}}}\right] \leqslant \sum_{i=0}^{p}\left[\frac{t_{l_{j}-(p+1)}}{t_{l_{j}}}\left(\rho_{\varphi}\left(\frac{1}{2}\right)\right)^{p+1-i}\right] \\
& \leqslant \sum_{i=0}^{p}\left(\rho_{\varphi}\left(\frac{1}{2}\right)\right)^{p+1-i}\left[\frac{t_{l_{j}-(p+1)}}{t_{l_{j}}}\right]=\bar{m}_{p+1}(j) \sum_{i=0}^{p}\left(\rho_{\varphi}\left(\frac{1}{2}\right)\right)^{p+1-i} \\
& =\bar{m}_{p+1}(j) \sum_{i=0}^{p}\left(\rho_{\varphi}\left(\frac{1}{2}\right)\right)^{1+p} \leqslant \bar{m}_{p+1}(j) \frac{\rho_{\varphi}\left(\frac{1}{2}\right)}{1-\rho_{\varphi}\left(\frac{1}{2}\right)}
\end{aligned}
$$

Поэтому можно положить

$$
c_{0}=\frac{\rho_{\varphi}\left(\frac{1}{2}\right)}{1-\rho_{\varphi}\left(\frac{1}{2}\right)} .
$$


Используя $l_{j}$ и набор $\bar{m}(j)$, согласно $(9),(10)$ определим $k_{j}$ и построим набор $m(j)$. Отметим, что для всех $j$ и всех $i=0,1, \ldots, l_{j}$ справедливо соотношение

$$
\frac{m_{i}(j)}{2^{l_{j}-i} m_{l_{j}}(j)} \leqslant 2 \rho_{\varphi^{-1}}\left(8+3 c_{0}\right) \varphi^{-1}\left(\frac{m_{i}(j)}{m_{l_{j}}(j)}\right)
$$

которое вытекает из леммы 3 и следующей цепочки неравенств:

$$
\begin{aligned}
\frac{m_{i}(j)}{2^{l_{j}-i} m_{l_{j}}(j)} & \leqslant \frac{\bar{m}_{i}(j)}{2^{l_{j}-i} \bar{m}_{l_{j}}(j)}=\frac{\left[\frac{t_{l_{j}-i}}{t_{l_{j}}}\right]}{2^{l_{j}-i}\left[\frac{t_{0}}{t_{l_{j}}}\right]} \leqslant \frac{2 t_{l_{j}-i}}{2^{l_{j}-i}}=2 \varphi^{-1}\left(t_{l_{j}-i}\right) \\
& =2 \varphi^{-1}\left(\frac{\frac{t_{l_{j}-i}}{t_{l_{j}}}}{\frac{t_{0}}{t_{l_{j}}}}\right) \leqslant 2 \varphi^{-1}\left(\frac{2\left[\frac{t_{l_{j}-i}}{t_{l_{j}}}\right]}{\left[\frac{t_{0}}{t_{l_{j}-i}}\right]}\right)=2 \varphi^{-1}\left(2 \frac{\bar{m}_{i}(j)}{\bar{m}_{l_{j}}(j)}\right) \\
& \leqslant 2 \varphi^{-1}\left(\left(4+\frac{3}{2} c_{0}\right) \frac{2 m_{i}(j)}{m_{l_{j}}(j)}\right) \leqslant 2 \rho_{\varphi^{-1}}\left(8+3 c_{0}\right) \varphi^{-1}\left(\frac{m_{i}(j)}{m_{l_{j}}(j)}\right) .
\end{aligned}
$$

Отсюда следует, что константу $c_{2}$ в лемме 9 можно взять в виде

$$
c_{2}=2 \rho_{\varphi^{-1}}\left(8+3 \frac{\rho_{\varphi}\left(\frac{1}{2}\right)}{1-\rho_{\varphi}\left(\frac{1}{2}\right)}\right) .
$$

По последовательности $\left\{k_{j}\right\}$ построим последовательность $\left\{n_{j}\right\}$. Для этого положим $n_{1}=2$, а остальные члены последовательности $\left\{n_{j}\right\}$ определим с помощью соотношений

$$
n_{j+1}=2^{k_{j}} n_{j}, \quad j=1,2, \ldots .
$$

По каждой паре чисел $n_{j}, l_{j}$ и набору $m(j)=\left\{m_{0}(j), m_{1}(j), \ldots, m_{l_{j}}(j)\right\}$ с помошью основной конструкции на квадрате $Q_{0}$ построим набор множеств $U_{n_{j}, l_{j}, m(j)}$, функцию $f_{n_{j}, l_{j}, m(j)}(\tau)$ и максимальный оператор $M_{n_{j}, l_{j}, m(j)}$. Определим дифференциальный ВF-базис $\mathbf{B}_{\varphi}$ с помощью равенства

$$
\mathbf{B}_{\varphi}=\bigcup_{j=1}^{\infty} U_{n_{j}, l_{j}, m(j)} .
$$

Покажем, что построенный таким образом базис удовлетворяет всем условиям теоремы.

Из леммы 4 следует, что для почти всех точек $t$ квадрата $Q_{0}$ найдется последовательность $B_{k}(t) \in \mathbf{B}_{\varphi}$ такая, что $t \in B_{k}(t)$ при всех $k$ и $\operatorname{diam} B_{k}(t) \rightarrow 0$ при $k \rightarrow \infty$.

Покажем, что дифференциальный базис $\mathbf{B}_{\varphi}$ дифференцирует пространство Лоренца $\Lambda(\varphi)$. Пусть $f \in \Lambda(\varphi), f \geqslant 0$ почти всюду. Зафиксируем $\alpha>0$ и положим

$$
D(f, \alpha)=\left\{\tau: M_{\mathbf{B}_{\varphi}} f(\tau)>\alpha\right\}, \quad D_{j}(f, \alpha)=\left\{\tau: M_{n_{j}, l_{j}, m(j)} f(\tau)>\alpha\right\} .
$$


Тогда справедливо равенство

$$
D(f, \alpha)=\bigcup_{j=1}^{\infty} D_{j}(f, \alpha)
$$

Из условия (38) следует, что при $j<i$ для любых двух элементов $U_{1} \in U_{n_{j}, l_{j}, m(j)}$, $U_{2} \in U_{n_{i}, l_{i}, m(i)}$ справедливо одно из двух соотношений: либо $U_{1} \cap U_{2}=U_{2}$, либо $U_{1} \cap U_{2}=\varnothing$. Поэтому если $U_{1} \subseteq D_{j}(f, \alpha), U_{2} \subseteq D_{i}(f, \alpha)$ и $U_{1} \cap U_{2}=U_{2}$, то множество $U_{2}$ можно исключить из $D_{i}(f, \alpha)$ и равенство $(40)$ сохранится. Отсюда следует, что можно считать выполненными соотношения $D_{i}(f, \alpha) \cap D_{j}(f, \alpha)=\varnothing$, $i \neq j$. Рассмотрим множество $D_{j}(f, \alpha)$ подробнее.

Согласно формуле (24) положим

$$
D_{j, s}(f, \alpha)=\left\{\tau: M_{n_{j}, l_{j}, m(j)} f(\tau) \chi\left(Q_{n_{j}}(s)\right)>\alpha\right\} .
$$

Тогда из (24) следует, что множества $D_{j, s}(f, \alpha)$ при разных $s$ не пересекаются, $D_{j, s}(f, \alpha) \subseteq Q_{n_{j}}(s)$ и

$$
D_{j}(f, \alpha)=\bigcup_{s=1}^{n_{j}^{2}} D_{j, s}(f, \alpha)
$$

Поэтому

$$
D(f, \alpha)=\bigcup_{j=1}^{\infty} \bigcup_{s=1}^{n_{j}^{2}} D_{j, s}(f, \alpha)
$$

и по-прежнему можно считать множества $D_{j, s}(f, \alpha)$ непересекаюшимися при разных значениях параметров. Для оценки меры каждого из множеств $D_{j, s}(f, \alpha)$ воспользуемся леммой 6 . Тогда получим

$$
\mu\left(D_{j, s}(f, \alpha)\right) \leqslant \frac{1}{\alpha} \int_{D_{j, s}(f, \alpha)} f(\tau) \xi_{j, s}(f, \alpha, \tau) d \tau .
$$

Рассмотрим функцию

$$
\xi_{\alpha}(\tau)=\sum_{j=1}^{\infty} \sum_{s=1}^{n_{j}^{2}} \chi\left(D_{j, s}(f, \alpha)\right) \xi_{j, s}(f, \alpha, \tau)
$$

Пусть $m \in \mathbb{Z}$. Оценим меру множества $\mu\left(\left\{\tau: \xi_{\alpha}(\tau) \geqslant 2^{m}\right\}\right)$. Поскольку множества $D_{j, s}(f, \alpha)$ не пересекаются, то

$$
\mu\left(\left\{\tau: \xi_{\alpha}(\tau) \geqslant 2^{m}\right\}\right)=\sum_{j=1}^{\infty} \sum_{s=1}^{n_{j}^{2}} \mu\left(\left\{\tau: \xi_{j, s}(f, \alpha, \tau) \chi\left(D_{j, s}(f, \alpha)\right) \geqslant 2^{m}\right\}\right) .
$$

Для оценки $\mu\left(\left\{\tau: \xi_{j, s}(f, \alpha, \tau) \chi\left(D_{j, s}(f, \alpha)\right) \geqslant 2^{m}\right\}\right)$ воспользуемся леммой 6 . При разных значениях параметра $m$ будем иметь 
1) если $m=-1,-2,-3, \ldots$, то

$$
\mu\left(\left\{\tau: \xi_{j, s}(f, \alpha, \tau) \chi\left(D_{j, s}(f, \alpha)\right) \geqslant 2^{m}\right\}\right) \leqslant \mu\left(D_{j, s}(f, \alpha)\right) ;
$$

2) если $m=0,1, \ldots, l_{j}$, то

$$
\mu\left(\left\{\tau: \xi_{j, s}(f, \alpha, \tau) \chi\left(D_{j, s}(f, \alpha)\right) \geqslant 2^{m}\right\}\right) \leqslant\left(1+c_{0}\right) \mu\left(D_{j, s}(f, \alpha)\right) \frac{m_{l_{j}-m}(j)}{m_{l_{j}}(j) 2^{m}} ;
$$

3) если $m=l_{j}+1, l_{j}+2, \ldots$, то

$$
\mu\left(\left\{\tau: \xi_{j, s}(f, \alpha, \tau) \chi\left(D_{j, s}(f, \alpha)\right) \geqslant 2^{m}\right\}\right)=0 .
$$

Учитывая тот факт, что набор $m(j)$ определен с помошью формулы (36), из (41) получим

$$
\begin{aligned}
\mu\left(\tau: \xi_{j, s}(\tau) \chi\left(D_{j, s}(f, \alpha)\right) \geqslant 2^{m}\right) & \leqslant 2\left(1+c_{0}\right) \mu\left(D_{j, s}(f, \alpha)\right) \frac{t_{m}}{2^{m} t_{0}} \\
& \leqslant 2\left(1+c_{0}\right) \mu\left(D_{j, s}(f, \alpha)\right) \frac{t_{m}}{2^{m}} .
\end{aligned}
$$

Здесь мы воспользовались тем фактом, что для любого $a \geqslant 1$ выполняется неравенство $[a] \leqslant a \leqslant 2[a]$, и тем, что все $m_{i}(j) \geqslant 1$.

Из последней формулы, используя вогнутость функции $\tilde{\varphi}$, получим

$$
\begin{aligned}
\sup _{m \geqslant 1} 2^{m} \tilde{\varphi}\left(\left\{\tau: \sum_{j=1}^{\infty} \sum_{s=1}^{n_{j}^{2}} \xi_{j, s}(f, \alpha, \tau) \chi\left(D_{j, s}(f, \alpha)\right) \geqslant 2^{m}\right\}\right) \\
\leqslant \sup _{m \geqslant 1} 2^{m} \tilde{\varphi}\left(\left(1+c_{0}\right) \frac{t_{m}}{2^{m}} \sum_{j=1}^{\infty} \sum_{s=1}^{n_{j}^{2}} \mu\left(D_{j, s}(f, \alpha)\right)\right) \\
\leqslant \sup _{m \geqslant 1} 2^{m} \tilde{\varphi}\left(\left(1+c_{0}\right) \frac{t_{m}}{2^{m}} \mu(D(f, \alpha))\right) \\
\leqslant\left(1+c_{0}\right) \sup _{m \geqslant 1} 2^{m} \tilde{\varphi}\left(\frac{t_{m}}{2^{m}} \mu(D(f, \alpha))\right) \\
\leqslant\left(1+c_{0}\right) \sup _{m \geqslant 1} 2^{m} \tilde{\varphi}\left(\frac{t_{m}}{2^{m}}\right) \rho_{\tilde{\varphi}}(\mu(D(f, \alpha))) \\
=\left(1+c_{0}\right) \rho_{\tilde{\varphi}}(\mu(D(f, \alpha))) \sup _{m \geqslant 1} 2^{m} \tilde{\varphi}\left(\frac{t_{m}}{2^{m}}\right) .
\end{aligned}
$$

Из равенства (4), лемм 1,2 , условия (3) с учетом (42) получим соотношение

$$
\begin{aligned}
& \mu(D(f, \alpha)) \leqslant \frac{1}{\alpha}\left\|f\left|\Lambda(\varphi)\|\| \sum_{j=1}^{\infty} \sum_{s=1}^{n_{j}^{2}} \chi\left(D_{j, s}(f, \alpha)\right) \xi_{j, s}(f, \alpha, \tau)\right| M(\tilde{\varphi})\right\| \\
& \leqslant 2 \frac{c_{1}(\tilde{\varphi})}{\alpha}\|f \mid \Lambda(\varphi)\| \sup _{m \geqslant 1} 2^{m} \tilde{\varphi}\left(\left\{\tau: \sum_{j=1}^{\infty} \sum_{s=1}^{n_{j}^{2}} \chi\left(D_{j, s}(f, \alpha)\right) \xi_{j, s}(f, \alpha, \tau) \geqslant 2^{m}\right\}\right) \\
& \leqslant 2 \frac{\left(1+c_{0}\right) c_{1}(\tilde{\varphi})}{\alpha}\|f \mid \Lambda(\varphi)\| \rho_{\tilde{\varphi}}(\mu(D(f, \alpha))) \sup _{m \geqslant 1} 2^{m} \tilde{\varphi}\left(\frac{t_{m}}{2^{m}}\right) .
\end{aligned}
$$


Используя равенство $2^{m} \tilde{\varphi}\left(\frac{t_{m}}{2^{m}}\right)=1$, которое следует из (28) и определения функции $\tilde{\varphi}$, прийдем к неравенству

$$
\frac{\mu(D(f, \alpha))}{\rho_{\tilde{\varphi}}(\mu(D(f, \alpha)))} \leqslant 2 \frac{\left(1+c_{0}\right) c_{1}(\tilde{\varphi})}{\alpha}\|f \mid \Lambda(\varphi)\| .
$$

Справедливо равенство $\rho_{\tilde{\varphi}}(\tau)=\tau \rho_{\varphi}\left(\frac{1}{\tau}\right)$ для функций растяжения функций $\tilde{\varphi}$ и $\varphi$. Поэтому окончательно получим

$$
\frac{1}{\rho_{\varphi}\left(\frac{1}{\mu(D(f, \alpha))}\right)} \leqslant 2 \frac{\left(1+c_{0}\right) c_{1}(\tilde{\varphi})}{\alpha}\|f \mid \Lambda(\varphi)\| .
$$

Из соотношения $(3)$ и очевидного неравенства $\rho_{\varphi}(\tau) \geqslant \frac{1}{\varphi\left(\frac{1}{\tau}\right)}$ следует, что

$$
\lim _{\lambda \rightarrow \infty} \inf \left\{t: \rho_{\varphi}(\lambda) \geqslant t\right\}=\infty
$$

Поэтому, применяя неравенство (43) в случае, когда $f(\tau)$ является характеристической функцией некоторого множества $\widetilde{D} \subset Q_{0}$, получим, что для любого $\alpha>0$ верно равенство

$$
\lim _{\mu(\widetilde{D}) \rightarrow 0} \mu(D(\chi(\widetilde{D}), \alpha))=0 .
$$

Из теоремы А $\S 1$ следует, что построенный базис является плотностным.

Поскольку для любой функции $f \in \Lambda(\varphi)$ выполнено равенство

$$
\lim _{\mu(\widetilde{D}) \rightarrow 0}\|f \chi(\widetilde{D}) \mid \Lambda(\varphi)\|=0
$$

то из неравенства (43) получим, что для любого $\alpha>0$ верно равенство

$$
\lim _{\mu(\widetilde{D}) \rightarrow 0} \mu(D(f \chi(\widetilde{D}), \alpha))=0 .
$$

Поскольку ВF-базис $\mathbf{B}_{\varphi}$ является плотностньм, то, применяя теорему В из $\S 1$, получим, что $\mathbf{B}_{\varphi}$ дифференцирует любую функцию $f \in \Lambda(\varphi)$, т.е. $\mathbf{B}_{\varphi}$ дифференцирует пространство $\Lambda(\varphi)$.

Покажем теперь, что $\mathbf{B}_{\varphi}$ не дифференцирует пространство Орлича $L_{h_{\varphi}}$. Для этого рассмотрим функцию

$$
f(\tau)=\sum_{j=0}^{\infty} f_{n_{j}, l_{j}, m(j)}(\tau) .
$$

Полагая $c_{2}=2 \rho_{\varphi^{-1}}\left(8+3 \frac{\rho_{\varphi}\left(\frac{1}{2}\right)}{1-\rho_{\varphi}\left(\frac{1}{2}\right)}\right)$ в лемме 9, из условий $(34)$ и (35) получим, что выполняется неравенство

$$
\left\|f \mid L_{h_{\varphi}}\right\| \leqslant \sum_{j=0}^{\infty} \lambda_{j}<\frac{1-\rho_{\varphi}\left(\frac{1}{2}\right)}{2} .
$$


С другой стороны, для каждого множества $U \in U_{n_{j}, l_{j}, m(j)}$ согласно лемме 8 будем иметь

$$
\frac{1}{\mu(U)} \int_{U} f(\tau) d \tau \geqslant \frac{1}{\mu(U)} \int_{U} f_{n_{j}, l_{j}, m(j)}(\tau) d \tau \geqslant \frac{1}{1+c_{0}}=1-\rho_{\varphi}\left(\frac{1}{2}\right)
$$

Поэтому для почти всех $t \in Q_{0}$ будет выполнено неравенство

$$
\overline{D_{\mathbf{B}_{\varphi}}(f, t)} \geqslant 1-\rho_{\varphi}\left(\frac{1}{2}\right)
$$

Из соотношений (45) и (46) следует, что дифференциальный базис $\mathbf{B}_{\varphi}$ не дифференцирует функцию $f(\tau)$ и, следовательно, пространство Орлича $L_{h_{\varphi}}$. Теорема полностью доказана.

\section{§ 3. Проблема окаймления для дифференциальных базисов}

Теперь перейдем к проблеме окаймления для дифференциальных базисов. Сначала продемонстрируем позитивные результаты. Отметим, что аналог теоремы 2 для дифференциальных базисов, инвариантных относительно сдвига и гомотетии, имеется в [13].

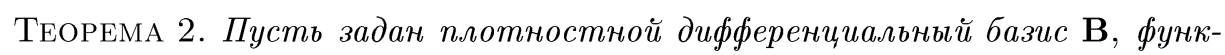
иия окаймления которого $h(\tau)=\psi_{\mathbf{B}}(\tau)$ принадлежит множеству $V$. По функиии $h \in V$ согласно (7) построим функцию $\varphi_{h} \in W$. Если $\varphi_{h} \in W$ удовлетворяет условию (3), то дифференциальньй базис $\mathbf{B}$ дифферениирует пространство $\Lambda\left(\varphi_{h}\right)$.

ДокАЗАТЕЛЬСтво. Обозначим

$$
\overline{\varphi_{h}}(\lambda)=\inf _{0<\tau \leqslant \min \left\{1, \frac{1}{\lambda}\right\}} \frac{\varphi_{h}(\lambda \tau)}{\varphi_{h}(\tau)}=\frac{1}{\rho_{\varphi_{h}}\left(\frac{1}{\lambda}\right)} .
$$

Покажем, что функция $\overline{\varphi_{h}}$ принадлежит $W$ и для нее выполняется неравенство

$$
\rho_{\overline{\varphi_{h}}}(2)=q_{0} \leqslant \rho_{\varphi_{h}}(2)<2
$$

Сначала отметим, что при каждом $\tau$ функция $\frac{\varphi_{h}(\lambda \tau)}{\varphi_{h}(\tau)}$ является вогнутой по $\lambda$. Поэтому и $\overline{\varphi_{h}}(\lambda)$ будет вогнутой функцией как нижняя грань вогнутых функций. Теперь убедимся, что выполнено соотношение (47). Действительно, по определению

$$
\rho \overline{\varphi_{h}}(2)=\sup _{0<\tau \leqslant \min \left\{1, \frac{1}{2}\right\}} \frac{\overline{\varphi_{h}}(2 \tau)}{\overline{\varphi_{h}}(\tau)}
$$

Для каждого фиксированного $\tau \in\left(0, \min \left\{1, \frac{1}{2}\right\}\right)$ и произвольного $\varepsilon>0$ выберем сначала точку $s_{\tau}$ так, чтобы

$$
\overline{\varphi_{h}}(\tau)=\inf _{0<s \leqslant \min \left\{1, \frac{1}{\tau}\right\}} \frac{\varphi_{h}(\tau s)}{\varphi_{h}(s)}>(1-\varepsilon) \frac{\varphi_{h}\left(\tau s_{\tau}\right)}{\varphi_{h}\left(s_{\tau}\right)} .
$$


Тогда придем к неравенству

$$
\begin{aligned}
\rho_{\overline{\varphi_{h}}}(2) & =\sup _{0<\tau \leqslant \min \left\{1, \frac{1}{2}\right\}} \frac{\overline{\varphi_{h}}(2 \tau)}{\overline{\varphi_{h}}(\tau)} \leqslant \sup _{0<\tau \leqslant \min \left\{1, \frac{1}{2}\right\}} \frac{\frac{\varphi_{h}\left(2 \tau s_{\tau}\right)}{\varphi_{h}\left(s_{\tau}\right)}}{(1-\varepsilon) \frac{\varphi_{h}\left(\tau s_{\tau}\right)}{\varphi_{h}\left(s_{\tau}\right)}} \\
& =\sup _{0<\tau \leqslant \min \left\{1, \frac{1}{2}\right\}} \frac{1}{1-\varepsilon} \frac{\varphi_{h}\left(2 \tau s_{\tau}\right)}{\varphi_{h}\left(\tau s_{\tau}\right)} \leqslant \frac{1}{1-\varepsilon} \rho_{\varphi_{h}}(2) .
\end{aligned}
$$

В силу произвольности $\varepsilon$ неравенство (47) доказано. Соотношение

$$
\lim _{\lambda \rightarrow 0} \frac{\overline{\varphi_{h}}(\lambda)}{\lambda}=\infty
$$

следует из (47) и неравенства

$$
\frac{\overline{\varphi_{h}}(\lambda)}{\lambda} \leqslant \frac{\overline{\varphi_{h}}\left(2^{-k} 2^{k} \lambda\right)}{\lambda} \leqslant \frac{\left(\rho \overline{\varphi_{h}}(2)\right)^{k} \overline{\varphi_{h}}\left(2^{-k} \lambda\right)}{\lambda} \leqslant \frac{\overline{\varphi_{h}}\left(2^{-k} \lambda\right)}{2^{-k} \lambda}\left(\frac{\rho \overline{\varphi_{h}}(2)}{2}\right)^{k} .
$$

Перепишем соотношение, определяюшее функцию окаймления, с учетом связи функций $\varphi_{h}$ и $h$ в следуюшем виде:

$$
\begin{aligned}
\varphi_{h}(\mu(D)) & \geqslant \varphi_{h}\left(\frac{\mu\left(\left\{\tau \in Q_{0}: M_{\mathbf{B}} \chi(D)(\tau)>\lambda\right\}\right)}{h\left(\frac{1}{\lambda}\right)}\right) \\
& \geqslant \varphi_{h}\left(\frac{1}{h\left(\frac{1}{\lambda}\right)}\right) \overline{\varphi_{h}}\left(\mu\left(\left\{\tau \in Q_{0}: M_{\mathbf{B}} \chi(D)(\tau)>\lambda\right\}\right)\right) \\
& =\lambda \overline{\varphi_{h}}\left(\mu\left(\left\{\tau \in Q_{0}: M_{\mathbf{B}}(\tau)>\lambda\right\}\right)\right) .
\end{aligned}
$$

Поскольку $\overline{\varphi_{h}} \in W$ и вьполнено (47), то, используя лемму 1 , соотношение (48) можно трактовать как выполнение для всех характеристических функций квадрата $Q_{0}$ неравенства

$$
\left\|M_{\mathbf{B}} \chi(D)\left|M\left(\overline{\varphi_{h}}\right)\left\|\leqslant c\left(\overline{\varphi_{h}}\right)\right\| \chi(D)\right| \Lambda(\varphi)\right\| .
$$

Согласно лемме А из $§ 1$ условие (49) влечет, что для любой функции $f \in \Lambda(\varphi)$ выполнено неравенство

$$
\left\|M_{\mathbf{B}} f\left|M\left(\overline{\varphi_{h}}\right)\left\|\leqslant 2 c\left(\overline{\varphi_{h}}\right)\right\| f\right| \Lambda(\varphi)\right\|,
$$

или

$$
\overline{\varphi_{h}}\left(\mu\left(\left\{\tau \in Q_{0}: M_{\mathbf{B}} f(\tau)>\lambda\right\}\right)\right) \leqslant \frac{c_{1}}{\lambda}\|f \mid \Lambda(\varphi)\| .
$$

Применяя последнее неравенство для функции $f \chi(\widetilde{D})$ и используя (44) и теорему В из $\S 1$, получим, что дифференциальньй базис $\mathbf{B}$ дифференцирует функцию $f \in$ $\Lambda(\varphi)$ и, следовательно, все пространство $\Lambda(\varphi)$. Теорема доказана.

Теперь покажем, что для каждого $p \in(1, \infty)$ существует дифференциальный базис $\mathbf{B}_{p}$, для которого функция окаймления при $\alpha \geqslant 1$ эквивалентна функции $\alpha^{p}$ и который не дифференцирует пространство Лебега $L^{p}$. 
Лемма 11. Пусть задано $1<p<\infty$. Пусть построенный по дифференииальному базису В оператор максимальной функиии $M_{\mathbf{B}}$ при всех $f \in \Lambda^{1 / p} u$ $\alpha>0$ удовлетворяет неравенству

$$
\mu\left(\left\{\tau: M_{\mathbf{B}} f(\tau)>\frac{1}{\alpha}\right\}\right)^{1 / p} \leqslant c \alpha\left\|f \mid \Lambda^{1 / p}\right\|
$$

с константой $c$, не зависящей от $f \in \Lambda^{1 / p}$ и $\alpha>0$.

Тогда при $\alpha \geqslant 1$ для функции окаймления дифференциального базиса справедлива оценка сверху

$$
\psi_{\mathbf{B}}(\alpha) \leqslant(c \alpha)^{p}
$$

$\left(\Lambda^{1 / p}=\Lambda(\varphi)\right.$, əде $\left.\varphi(\tau)=\tau^{1 / p}\right)$.

ДокАЗАТЕЛЬСТво. Применим неравенство $(50)$ для функции $\chi(D)$. Тогда получим

$$
\mu\left(\left\{\tau: M_{\mathbf{B}} \chi(D)(\tau)>\frac{1}{\alpha}\right\}\right)^{1 / p} \leqslant c \alpha \mu(D)^{1 / p}
$$

или

$$
\mu\left(\left\{\tau: M_{\mathbf{B}} \chi(D)(\tau)>\frac{1}{\alpha}\right\}\right) \leqslant(c \alpha)^{p} \mu(D) .
$$

Последнее неравенство и доказывает лемму.

Теорема 3. Пусть задано $p \in(1, \infty)$. Тогда существует дифференциальный ВF-базис $\mathbf{B}_{p}$, функция окаймления которого при $\alpha \geqslant 1$ әквивалентна $\alpha^{p}$, но этот дифференциальный базис не дифференцирует пространство Лебега $L^{p}$.

ДоКАЗАТЕльство. Для построения дифференциального базиса $\mathbf{B}_{p}$ применим конструкцию базиса $\mathbf{B}_{\boldsymbol{\varphi}}$ из теоремы 1 , определяя конкретные параметры для заданного числа $p \in(1, \infty)$.

Итак, зафиксируем число $p \in(1, \infty)$. Тогда $\varphi(\tau)=\tau^{1 / p}, \rho_{\varphi}(\lambda)=\lambda^{1 / p}$, $\varphi^{-1}(\tau)=\tau^{p}, \rho_{\varphi^{-1}}(\lambda)=\lambda^{p}, c_{0}=\frac{1}{2^{1 / p}-1}$.

Положим $\lambda_{j}=\left(2^{-\gamma_{0}}\right)^{j}$. Тогда можно выбрать $\gamma_{0}>0$ так, чтобы для последовательности $\left\{\lambda_{j}\right\}_{j=1}^{\infty}$ вьполнялось условие (34). Затем определим последовательность $\left\{l_{j}\right\}_{j=1}^{\infty}$ равенством

$$
l_{j}=\left[\left(2\left(8+3 c_{0}\right)^{p} \lambda_{j}^{p}\right)^{\frac{1}{1-p}}\right]
$$

$[x]$ - целая часть $x$.

Отметим, что из определения $\lambda_{j}$ и (51) следует справедливость эквивалентности

$$
l_{j} \simeq\left(2^{\gamma_{1}}\right)^{j},
$$

где $\gamma_{1}=\frac{\gamma_{0} p}{p-1}$.

Решая уравнение (28), нетрудно видеть, что верно равенство

$$
t_{i}=2^{\frac{i}{1-p}}
$$


Поэтому для каждого $j \in \mathbb{N}$ набор $\bar{m}(j)$ можно определить с помощью соотношения

$$
\bar{m}_{i}(j)=\left[\frac{t_{l_{j}-i}}{t_{l_{j}}}\right]=\left[2^{\frac{i}{p-1}}\right], \quad i=0,1, \ldots, l_{j} .
$$

По $l_{j} \in \mathbb{N}$ и наборам $\bar{m}(j)$ с помощью $(9),(10)$ построим $k_{j}, n_{j} \in \mathbb{N}$ и наборы $m(j)$.

Из (53) следует, что найдется абсолютная константа $c_{3}>0$ такая, что для каждого $j \in \mathbb{N}$ выполнено соотношение

$$
c_{3} 2^{\frac{l_{j}-i}{1-p}} \leqslant \frac{m_{i}(j)}{m_{l_{j}}(j)} \leqslant 2^{\frac{l_{j}-i}{1-p}}, \quad i=1,2, \ldots, l_{j} .
$$

По параметрам $n_{j}, l_{j}, m(j)$ построим наборы множеств $U_{n_{j}, l_{j}, m(j)}$ и дифференциальный базис $\mathbf{B}_{p}$. Покажем, что построенный базис удовлетворяет утверждению теоремы.

Найдем оценку его функции окаймления. Из неравенства (43) и леммы 11 получим, что при $\alpha \geqslant 1$ с некоторой константой $c_{4}$ выполнено соотношение

$$
\psi_{\mathbf{B}}(\alpha) \leqslant c_{4} \alpha^{p}
$$

Для получения оценки снизу используем множества $R_{n, l, m}(s)=\bigcup_{j=1}^{n^{2}} R_{U_{n}(j)}(s)$, $s=0,1, \ldots, l$. Тогда из соотношений $(26),(52)$ и (54) следует, что найдутся $j_{0} \in \mathbb{N}$ и константа $c_{5}>0$ такие, что при всех $j>j_{0}$ для каждого $s=0,1, \ldots, l_{j}$ выполняются неравенства

$$
\begin{gathered}
\mu\left(\left\{t: M_{\mathbf{B}_{p}} \chi\left(R_{n_{j}, l_{j}, m(j)}(s), t\right) \geqslant \frac{m_{s}(j)}{\left(1+c_{0}\right) m_{l_{j}}(j)}\right\}\right) \\
=\mu\left(\left\{t: \sup \left\{\frac { 1 } { \mu ( U ) } \int _ { U } \chi \left(R_{\left.n_{j}, l_{j}, m_{(j)}(s), \tau\right) d \tau:}\right.\right.\right.\right. \\
\left.\left.\left.t \in U \in \mathbf{B}_{p}\right\} \geqslant \frac{m_{s}(j)}{\left(1+c_{0}\right) m_{l_{j}}(j)}\right\}\right) \\
\geqslant\left(1-\frac{c_{0}}{l_{j}}\right) \frac{m_{l_{j}}(j) 2^{l_{j}-s}}{m_{s}(j)} \mu\left(R_{n_{j}, l_{j}, m(j)}(s)\right) \\
\geqslant c_{5}\left(\frac{m_{l_{j}}(j)}{m_{s}(j)}\right)^{p} \mu\left(R_{n_{j}, l_{j}, m(j)}(s)\right) .
\end{gathered}
$$

Из соотношений $(52)$ и (56) получим, что при $\alpha \geqslant 1$ с некоторой константой $c_{6}$ выполнено соотношение

$$
\psi_{\mathbf{B}}(\alpha) \geqslant c_{6} \alpha^{p}
$$

что дает оценку снизу функции окаймления.

Неравенства (55) и (57) показывают, что у построенного дифференциального базиса $\mathbf{B}_{p}$ при $\alpha \geqslant 1$ функция окаймления $\psi_{\mathbf{B}_{p}}(\alpha)$ ведет себя как $\alpha^{p}$. С другой стороны, согласно теореме 2 дифференциальньй базис $\mathbf{B}_{p}$ не дифференщирует пространство Лебега $L^{p}$. Теорема полностью доказана. 


\section{Список литературы}

1. Гусман M. Дифференцирование интегралов в $\mathbb{R}^{n}$. М.: Мир, 1978.

2. Stokolos A. M. On the differentiation of integrals of functions from $L \Phi(L) / /$ Studia Math. 1988. V. 88. P. 103-120.

3. Stokolos A. M. On the differentiation of integrals of functions from Orlicz classes // Studia Math. 1989. V. 94. P. 35-50.

4. Бережной Е.И. О дифференцировании интегралов от функций из симметричных пространств дифференциальными базисами // Analysis Mathematica. 1996. Т. 22. C. $267-288$.

5. Бережной E.И., Перфильев A.A. Различение симметричных пространств и $L^{\infty}$ с помощью дифференциального базиса // Матем. заметки. 2001. Т. 68. № 3. С. 515-523.

6. Lebesgue A. Sur l'integration des fonctions discontinues // Ann. Sci. Ecole Norm. Sup. 1910. V. 27. P. 361-450.

7. Jessen B., Marzinkiewicz J., Zygmund A. Note on the differentiality of multiple integrals // Fund. Math. 1935. V. 25. P. 217-234.

8. Zygmund A. A note on the differentiality of multiple integrals // Collog. Math. 1967. V. 16. P. 199-204.

9. Saks S. On the strong derivatives of functions of integrals // Fund. Math. 1935. V. 25. P. 235-252.

10. Melero B. A negative result in differentiation theory // Studia Math. 1982. V. 72. P. 173-182.

11. Крейн С.Г., Петунин Ю.И., Семенов Е. М. Интерполяция линейных операторов. М.: Наука, 1977.

12. Lindenstrauss J., Tzafriri L. Classical Banach spaces. I, II. Berlin: Springer, 1979.

13. Бережной Е. И. Дифференциальные свойства базисов и проблема окаймления для симметричных пространств // Сиб. матем. журн. 1995. Т. 36. № 6. С. 1234-1250.

Ярославский госуниверситет им. П. Г. Демидова.

E-mail: ber@uniyar.ac.ru

Поступило в редакцию

7.V.2001 